\title{
An Unusual Presentation of Leptospirosis: A Case Report
}

\author{
Monika Jawanjal, Smita Patil, Manish Pendse, Archana Bhate, Shariq Hooda \\ Padmashree Dr. D Y Patil Hospital and Research Centre, Mumbai, India \\ Email: drmonika257@gmail.com, archana_bhate@hotmail.com
}

Received 11 April 2014; revised 11 May 2014; accepted 12 June 2014

Copyright (C) 2014 by authors and Scientific Research Publishing Inc.

This work is licensed under the Creative Commons Attribution International License (CC BY). http://creativecommons.org/licenses/by/4.0/

c) (i) Open Access

\begin{abstract}
Leptospirosis is a life-threatening zoonotic disease of global distribution. It has variable presentation ranging from mild febrile illness to life-threatening complications like acute renal failure, acute hepatic failure, pulmonary hemorrhages and cardiac arrhythmias. Cardiac manifestations of leptospirosis are not uncommon but are underdiagnosed. Cardiac manifestations may vary from minor rhythm abnormalities to fatal ventricular arrhythmias. We report an unusual presentation of leptospirosis in a patient who presented to the Emergency Department with epigastric pain and congestive cardiac failure with rhythm disturbances and selective myocardial involvement mimicking as Non ST elevation Inferior wall myocardial infarction (NSTEMI). The Cardiac manifestations of Leptospirosis need meticulous intensive care management as the mortality rate is very high in this subset of patients. Index suspicion and early treatment remains the mainstay of this life threatening zoonotic disease.
\end{abstract}

\section{Keywords}

Leptospirosis, Congestive Cardiac Failure

\section{Introduction}

Leptospirosis is a potentially fatal zoonotic disease which is caused by the pathogenic spirochete of genus leptospira, known to cause multiorgan dysfunction including acute renal failure, severe rhabdomyolysis, hepatic dysfunction, pulmonary haemorrhages and myocarditis [1]. It is a disease of worldwide distribution. Human infection occurs either through the direct contact with infected animals or more of the times through the contact with water or soil contaminated with urine of infected rodents or animals. Cardiac manifestations of leptospirosis are not uncommon but are underdiagnosed [1].

We report an unusual presentation of leptospirosis in a patient who presented to the Emergency Department 
with epigastric pain and congestive cardiac failure with rhythm disturbances and selective myocardial involvement mimicking as Non ST elevation Inferior wall myocardial infarction (NSTEMI).

\section{Case Report}

A 53 years old male patient presented to the Emergency Department with complains of epigastric pain, waxing and waning since 12 to 14 hours and difficulty in breathing since 6 hours before presenting to us. On enquiry patient gave history of one fever spike a day prior.

On presentation, the pulse was 58 per minute and regular with blood pressure of 90/70 mm of $\mathrm{Hg}$. The clinical features were suggestive of congestive cardiac failure viz raised Jugular venous pressure, mild bilateral symmetrical pitting pedal oedema and Bilateral basal rales. Also the patient was icteric.

The ECG was done in the ER which was suggestive of junctional rhythm with $T$ wave inversions in inferior leads viz leads II, III and avF (see Figure 1). Cardiac enzymes and Xray Chest confirmed the diagnosis of Congestive cardiac failure (see Figure 2) and myocardial ischemia. Troponin T and Trop I were positive and BNP was 1044; significantly raised (Table 1).

The patient was immediately shifted to intensive care unit for further management and was put on BIPAP support. Within 1 hour of admission, the patient had one episode of fever of $101^{\circ} \mathrm{F}$. As the patient was from endemic area for Malaria, Dengue and Leptospirosis, we started the patient on injectable anti-malarials and Ceftrioxone along with Noradrenalin infusion for hypotension and Injectable diuretics for congestive cardiac failure. However, we decided not to start the heparin therapy for our patient before the complete blood count is available.

The Blood reports were as Table 1.

The 2D echocardiogram was done which confirmed the segmental hypokinesia of mid-basal inferior wall with left ventricular ejection fraction of $45 \%$ and mild pulmonary hypertention (PASP $=50 \mathrm{~mm}$ of $\mathrm{Hg}$ ). One episode of fever with thrombocytopenia needed further evaluation so we evaluated him for Malaria, Dengue and Leptospirosis which all turned out to be negative on day 1 of admission.

The EKG was repeated every 4 hourly which was suggestive of the junctional rhythm with $\mathrm{T}$ wave inversion in inferior leads.

On second day the patient's platelets decreased even more to $35,000 / \mathrm{mm}^{3}$ and the ECG changes remained the same. Heparin therapy could not be started because of thrombocytopenia and unavailability of single donor platelets. We continued treatment with anti-malarials, Ceftrioxone, Diuretics as well as intravenous fluid therapy in view of acute kidney injury.

On third day, the platelates improved marginally but the serum creatinine started decreasing and also the EKG showed normal sinus rhythm (see Figure 3). The patient started improving clinically and hemodynamically on fourth day. As it was a multiorgan involvement, we strongly suspected leptospirosis and so repeated the test for Leptospirosis antibodies again on day 4 which tuned out to be positive for both IgM and IgG (Table 1).

On the fifth day, there was significant improvement in the patient clinically and the patient was shifted out of Intensive care unit. However, as the patient primarily presented with Congestive cardiac failure with ischemic changes on EKG, so coronary angiography was done to rule out Coronary artery disease on ninth day which

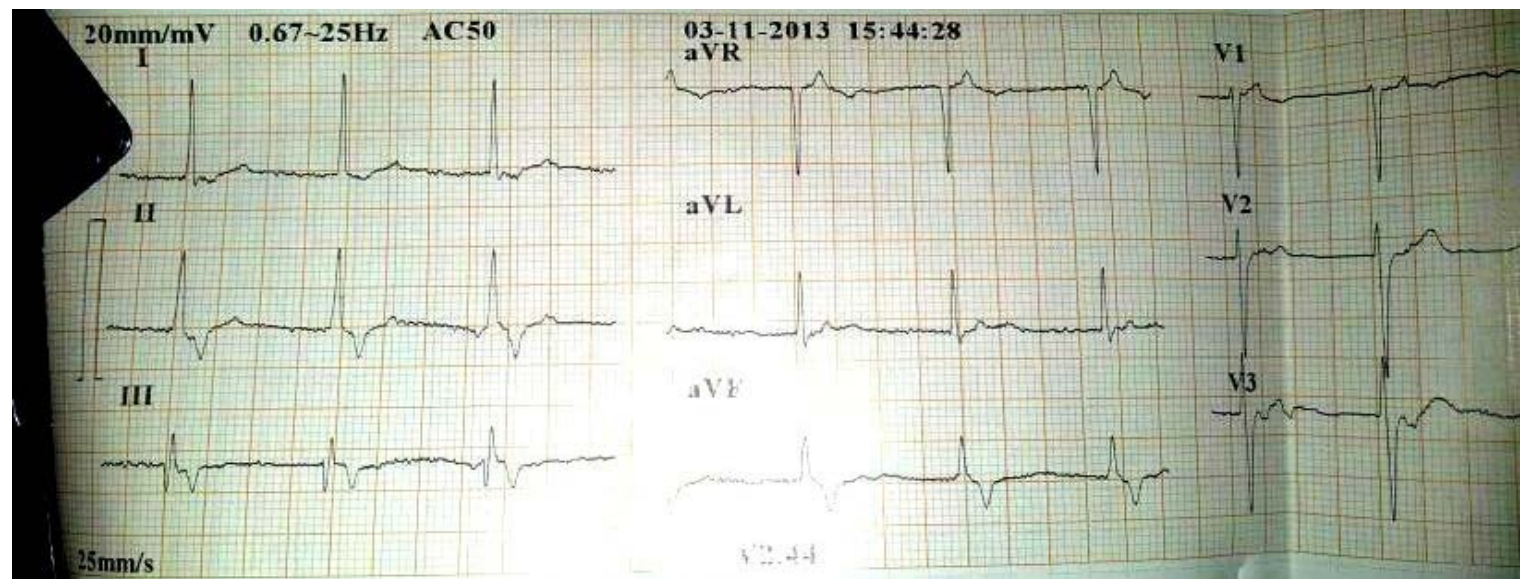

Figure 1. EKG on presentation. 


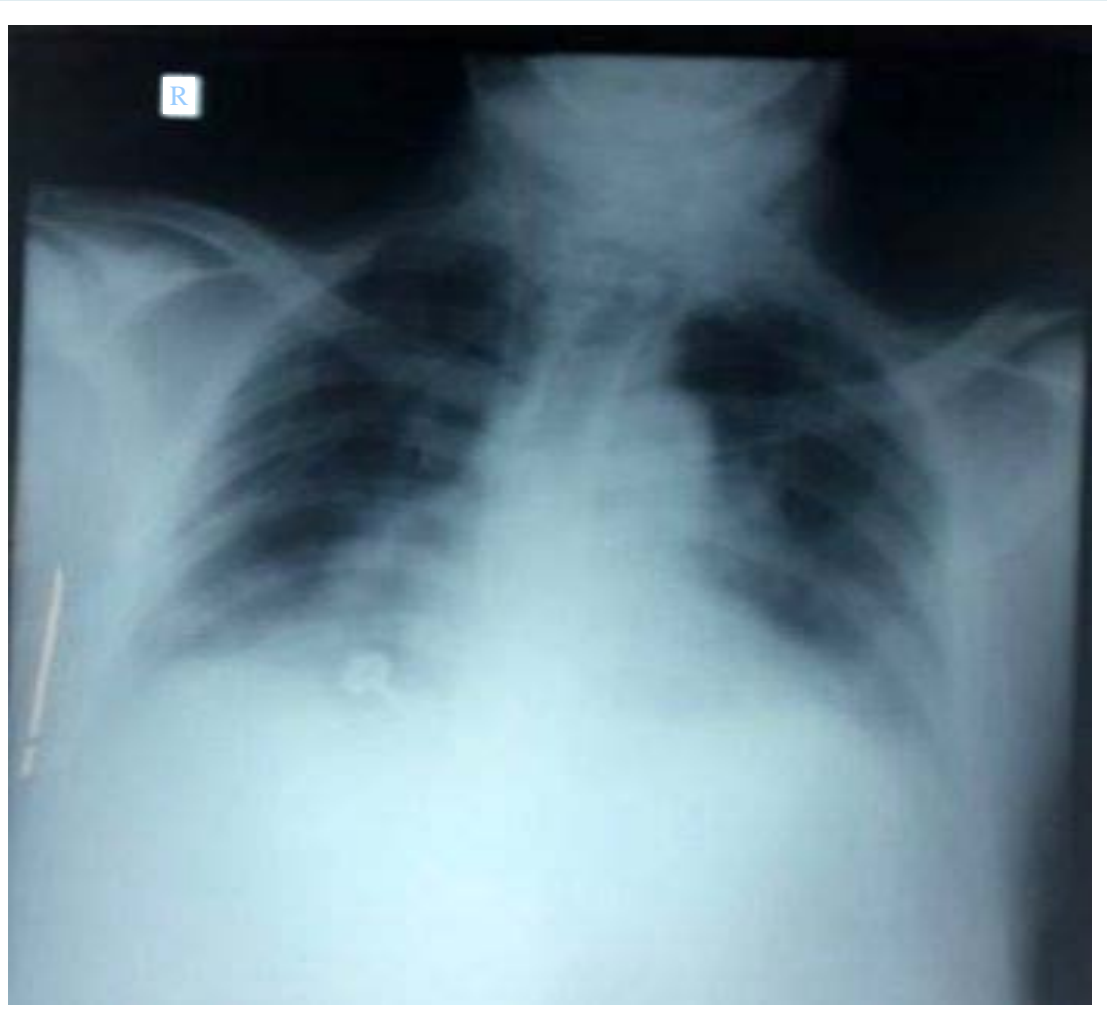

Figure 2. X ray chest AP view on presentation.

Table 1. Blood reports.

\begin{tabular}{|c|c|c|c|c|c|c|}
\hline & Day 1 & Day 2 & Day 3 & Day 4 & Day 5 & Day 7 \\
\hline $\mathrm{Hb}(\mathrm{gm} \%)$ & 12.2 & 11.0 & 11.3 & 11.4 & 12.3 & 12.4 \\
\hline TLC & 8100 & 12,000 & 9500 & 8600 & 8400 & 8300 \\
\hline Platelets & 39,000 & 34,000 & 35,000 & 66,000 & 90,000 & 124,000 \\
\hline Creatinine (mg\%) & 2.4 & 3.2 & 2.0 & 1.4 & 1.0 & 1.0 \\
\hline BUN & 34.4 & 40 & - & - & - & - \\
\hline $\mathrm{Na}^{+}$ & 126 & 129 & 130 & 131 & 130 & 133 \\
\hline $\mathrm{K}^{+}$ & 4.8 & 4.6 & 3.9 & 3.7 & 3.6 & 3.6 \\
\hline $\mathrm{Cl}^{-}$ & 99 & 100 & 100 & 100 & 101 & 101 \\
\hline Total Billirubin & 3.0 & 4.0 & - & 2.4 & 1.4 & 1.0 \\
\hline Direct & 0.4 & 0.8 & - & 0.4 & 0.4 & 0.2 \\
\hline Indirect & 2.6 & 3.2 & - & 2.0 & 1.0 & 0.8 \\
\hline AST & 167 & 324 & - & 110 & 88 & 58 \\
\hline ALT & 147 & 312 & - & 106 & 76 & 52 \\
\hline Rapid Malarial Antigen & Negative & - & - & - & - & - \\
\hline Rapid Dengue Antibodies Negative & Negative & - & - & - & Negative & - \\
\hline Leptospira & Negative & - & - & - & IgM-Positive & - \\
\hline Antibodies & & & & & IgG-Positive & \\
\hline
\end{tabular}




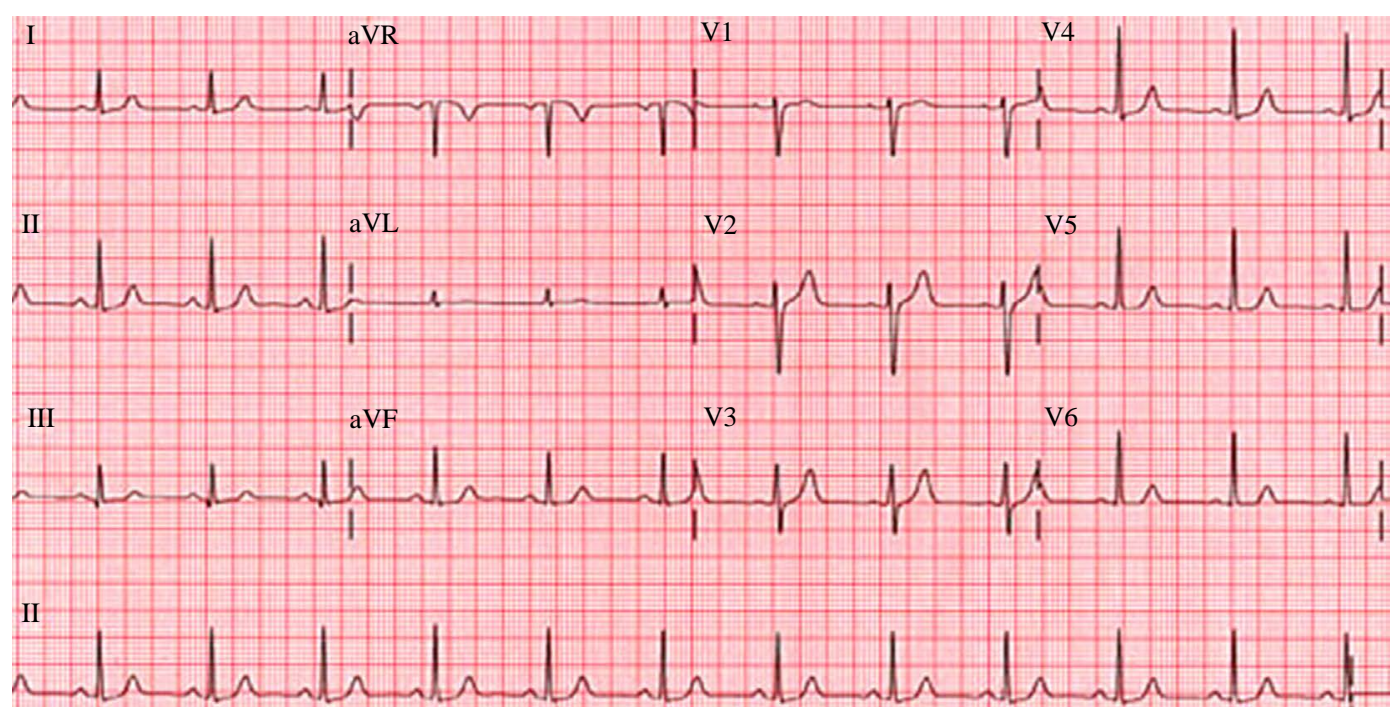

Figure 3. EKG after reverting to normal sinus rhythm.

confirmed normal coronaries.

A repeat 2D echocardiogram also showed normal Left ventricular ejection fraction of $60 \%$ without any regional wall motion abnormality or pulmonary hypertension. The patient was discharged on oral Cefixime 200 mg BID and Doxycycline 100 mg for 5 days.

\section{Discussion}

Leptospirosis was first reported around 100 years ago by Adolf Weil in Heidelberg [1]. Leptospirosis is endemic in South-Eastern Asia, where a lot of regions register the highest worldwide incidence of human leptospirosis [2].

Leptospirosis can occur at any time during the year, with a peak incidence during summer and autumn. An average incubation period is 14 days [1] [2]. Being aerobic bacteria responsible for ubiquitous zoonoses (rats, birds, amphibians), the Leptospira spirochetes can survive, after urinary excretion, for up to 9 months in their natural reservoir, sweet water [1]. Clinically, the presentation of Leptospirosis can vary from mild non specific flu-like symptoms to a severe disease with complications like acute renal failure, rhabdomyolysis, hepatic dysfunction, Myocarditis and conduction disturbances [1]-[3].

\section{Pathophysiology}

Leptospirosis is characterised by vasculitis endothelial damage and inflammation. Petechial hemorrhages are seen [1] [4]. In kidneys interstitial nephritis is a major finding along with cellular infiltration with neutrophils and monocytes. Leptospira can be seen in renal tubules. Spirochetes can be found in the walls of capillaries, medium and large vessels [1] [5].

CNS manifestation can be in the form of asceptic meningitis [1] [6].

Hepatic dysfunction is in the form of intrahepatic cholestasis and hypertrophy of kupffer cells [1] [5].

In lungs, alveolar hemorrhages are common.

Cardiovascular involvement in leptospirosis is common but may be underestimated. Cardiac menifestations are variable ranging from rhythm disturbances, myocarditis, myopericarditis to fatal cardiac arrhythmias. Congestive cardiac failure is rarely seen in leptospirosis.

Rhythm disturbances are seen in as much as 70\% of the patients [1] [7].

Severe cardiac involvement is associated with mortality rate of as much as 54\%[1] [8] [9]. There is interstitial myocarditis and infiltration of lymphocytes and plasma cells which lead to cardiac menifestations of leptospirosis.

The segmental involvement of myocardium suggests coronary arteritis which mimic NSTEMI [8] [9]. In our case, there was segmental involvement of inferior wall confirmed by 2D Echocardiography. 
However we needed to rule out coronary artery disease to confirm the coronary arteritis due to leptospirosis. The Coronary angiography was done on ninth day of admission which showed normal coronaries.

ELISA IgMon fourth day of illness can be confirmatory. The treatment of Leptospirosis includes Penicillin G 1.5 MU IV qid or Ceftrioxone $1 \mathrm{gm}$ IV OD [1] [10].

Oral Doxycyclin (200 mg) once a week can be used as a prophylaxis in endemic areas [1] [10]. Patients with renal failure may require dialysis. Corticosteroid therapy with high-dose pulsed methylprednisolone $(30 \mathrm{mg} / \mathrm{kg} / \mathrm{d}$, not to exceed $1500 \mathrm{mg}$ has also shown improvement in patients with severe disease with renal failure and Pulmonary haemorrhages [5].

We gave Injectable Ceftrioxone 2 gm Intravenous once a day for 7 days. The patient improved dramatically and was discharged after a normal coronary angiogram.

\section{Conclusion}

Leptospirosis is a life threatening zoonotic disease with varied presentation. In endemic area, fever with renal dysfunction with rhythm abnormalities Leptospirosis should be suspected. Congestive cardiac failure with segmental myocardial involvement and rhythm disturbances is a rare presentation in leptospirosis and is associated with very high mortality rate. Timely diagnosis and meticulous intensive care management and appropriate antibiotic therapy remain the mainstay of the treatment. Appropriate measures for prevention and control of this life threatening disease should target the rodent reservoirs, domestic animal carriers and environmental measures in the endemic areas. Prophylaxis with Doxycycline should be used in the endemic areas.

\section{References}

[1] Paul, N.V., et al. (2001) Leptospira Species (Leptospirosis). Infectious Diseases and Their Etiologic Agents.

[2] Vinetz, J.M. (2001) Leptospirosis. Current Opinion in Infectious Diseases, 14, 527-538. http://dx.doi.org/10.1097/00001432-200110000-00005

[3] Leblebicioglu, H., Sencan, I., Sunbul, M., et al. (1996) Weil’s Disease: Report of 12 Cases. Scandinavian Journal of Infectious Diseases, 28, 637-639. http://dx.doi.org/10.3109/00365549609037976

[4] Henry, W.L., De Maria, A., Gramiak Henry, W.L., et al. (1980) Report of the American Society of Echocardiography Committee on Nomenclature and Standards in Two-Dimensional Echocardiography. Circulation, 62, 212-217. http://dx.doi.org/10.1161/01.CIR.62.2.212

[5] Levett, P.N. (2001) Leptospirosis. Clinical Microbiology Reviews, 14, 296-326. http://dx.doi.org/10.1128/CMR.14.2.296-326.2001

[6] Rajajee, S., Shankar, J. and Dhattatri, L. (2002) Pediatric Presentations of Leptospirosis. Indian Journal of Pediatrics, 69, 851-853.

[7] Ram, P. and Chandra, M.S. (1985) Unusual Electrocardiographic Abnormality in Leptospirosis: Case Reports. Angiology, 36, 477-482. http://dx.doi.org/10.1177/000331978503600711

[8] Vijayachari, P., Sinha, A., et al. (1999) Clinico-Epidemiological Study of Hospitalized Cases of Severe Leptospirosis. Indian Journal of Medical Research, 109, 94-99.

[9] Mel’nik, G.V., Degtiar, L.D. and Andreev, R.E. (1999) Cardiovascular Function in Leptospirosis Convalescents. Tekrapevticheskii Arkhiv, 71, 32-34.

[10] Artigou, J.Y., Chauvet, J.P., Clergue, F., et al. (1986) Acute Myocarditis Disclosing Leptospirosis. Annales de Cardiologie Angiologie (Paris), 35, 387-389. 